[Agr. Biol. Chem., Vol. 31, No. 12, p. 1441 1447, 1967]

\title{
Studies on the Chemical Regulation of Alkaloid Biosynthesis in Tobacco Plants
}

\author{
Part II. Inhibition of Alkaloid Biosynthesis \\ by Exogenous Auxins
}

\author{
By Norio Yasumatsu \\ Hatano Tobacco Experiment Station, Japan Monopoly \\ Corporation, Hatano-shi, Kanagawa-ken \\ Received July 3, 1967
}

\begin{abstract}
Effect of auxin treatment upon the alkaloid content of Nicotiana was examined using hydroponically grown plants. Nicotine content of Nicotiana tabacum was reduced by the addition of 3-indoleacetic acid or 2,4-dichlorophenoxyacetic acid to nutrient solution, and the incorporation of nitrate-15 $\mathrm{N}$ into nicotine was decreased by the addition of 2, 4-dichlorophenoxyacetic acid. Contents of nicotine, nornicotine, anabasine and anatabine in Nicotiana glutinosa were decreased by 2,4-dichlorophenoxyacetic acid added to the nutrient solution. Foliar applications of eleven kinds of synthetic auxins were effective in reducing nicotine content of Nicotiana tabacum and the effectiveness of the compounds seemed to be concerned with their auxin activities. Foliar application of auxin tended to increase the dry weight of stems whereas decreased that of roots, and, moreover, higher content of soluble sugars and lower content of starch in leaves were observed by the treatment. A possibility of a practical application of auxin to reduce the nicotine content of tobacco plants were drawn from this work.
\end{abstract}

It has been known that the nicotine content of tobacco plants is affected by enviromental and cultural conditions, such as soil moisture, amount of nitrogen supplied and topping (removal of flower buds and some leaves). Among these conditions, the most striking effect is brought about by topping, by which an increased accumulation of alkaloid in tobacco plants is observed. The reason why the topped plants accurnulate a larger amount of alkaloid has not yet been known. There

\footnotetext{
Abbreviations: IAA, 3-Indoleacetic acid; NAA, $\alpha$ Naphthaleneacetic acid; TBA, 2, 3,6-Trichlorobenzoic acid; 2, 4-D, 2, 4-Dichlorophenoxyacetic acid; 2, 4, 5T, 2, 4,5-Trichlorophenoxyacetic acid; CPA, 4-Chlorophenoxy acetic acid; MCP, 2-Methyl-4-chlorophenoxyacetic acid; 2, 4-DP, 2-(2, 4-Dichlorophenoxy) isopropionic acid; 2, 4, 5-TP, 2-(2, 4, 5-Trichlorophenoxy) isopropionic acid; MCPP, 2-(2-Methyl-4-chlorophenoxy) isopropionic acid; NOXA, $\beta$-Naphthoxyacetic acid.
}

seemed to exist some apical dominancy over the alkaloid biosynthesis in tobacco plants. Hasegawa ${ }^{11}$ has shown that nicotine of tobacco plants is produced in the roots. In the experiment using excised root culture of Nicotiana tabacum var. Turkish, Solt ${ }^{21}$ has found that there is a close parallelism between root growth and nicotine yield, and that reduced root growth caused by the treatment of IAA results in decrease of nicotine production. Paleg et al. $^{3 !}$ reported that high activity of endogenous auxin was observed in flowers of tobacco plants. It may be therefore possible

1) H. Hasegawa, Bot. Mag. (Tokyo), 51, 306 (1937).

2) M. L. Solt, Plant Physiol., 32, 480 (1957).

3) L. G. Paleg and R. M. Muir, Australian J. Biol. Sci., 12, 340 (1959). 
to suppose that the alkaloid biosynthesis in tobacco plants is influenced by the endogenous auxin. But there is no evidence to elucidate this supposition. The present work was undertaken to investigate the effect of exogenous auxins, which was sprayed to foliage or added to nutrient solutions, upon the alkaloid biosynthesis in Nicotiana.

\section{MATERIALS AND METHODS}

Synthetic auxins. IAA, NAA, TBA, 2, 4-D and 2, 4, 5-T were obtained commercially. NOXA, CPA, 2, 4-DP, 2, 4, 5-TP, MCP and MCPP were kindly supplied by Dr. M. Gotoh of the Toa Agricultural Chemicals Company. Derivatives of auxins were prepared in the following way. By the reaction of GPA and an excess of phosphorous trichloride, the acid chloride was obtained and dissolved in chloroform. The methylester, isopropylester, amide and methylamide of CPA were obtained by addition of absolute methanol, absolute isopropanol, $28 \%$ aqueous ammonia or $48 \%$ aqueous methylamine to the chloroform solution. The methylesters of 2,4-D and 2, 4, 5$\mathrm{T}$ were prepared in a similar manner.

Tobacco plant. Unless otherwise stated, Nicotiana tabacum var. Bright Yellow was used in this work. The plants were grown hydroponically in plastic pots containing 11 of nutrient solution ${ }^{4}$ in a greenhouse. When eighteen leaves developed the plants were decapitated leaving eleven leaves and used for auxin treatment. In another experiment Nicotiana glutinosa was grown in a similar way, decapitated leaving eighteen leaves when it has developed twentyone leaves and used for 2, 4-D treatment. Three plants were employed for each treatment.

Feeding of auxin. After the decapitation of tobacco plants auxin was added to nutrient solution and thereafter the solution containing auxin was exchanged every 4 days. For the application of auxin to foliage, aqueous auxin solution, which contained $0.1 \%$ of Tween 20 as a surfactant, was sprayed to upper surface of leaves from 5 to 6 p.m. at the beginning of treatment and no additional treatment was performed. In ordinary cases $20 \mathrm{ml}$ of the solution was sprayed to each plant. Unless otherwise stated, after beginning of the both auxin

4) T. Takahashi, Bull. Hatano Tobacco Expt. Sta., No. 50, 33 (1961). treatments the plants were allowed to grow following two weeks.

Feeding of ${ }^{15} \mathbf{N}$-labeled nitrate. Decapitated $N$. tabacum plants were pretreated with the nutrient solu. tion containing 1 p.p.m. of $2,4-\mathrm{D}$ for $24 \mathrm{hrs}$. and then transferred to $500-\mathrm{ml}$ beakers containing the nutrient solution having ${ }^{15} \mathrm{~N}$-labeled nitrate $(50$ p.p.m. as nitrogen) and 2, 4-D (1 p.p.m.). After the administration of nitrate- $15 \mathrm{~N}$ for $24 \mathrm{hrs}$. the plants were harvested and used for analysis. Labeled nitratenitrogen contained 38 atom per cent of ${ }^{15} \mathrm{~N}$.

Assay of tobaceo alkaloids. $N$. tabacum plants harvested were dried in a forced draft oven at $70^{\circ} \mathrm{C}$, ground in a Wiley mill and used for analysis. Alkaloid was separated by a steam-distillation with addition of an excess of sodium hydroxide and estimated spectrophotometrically.5) In a preliminary experiment no peaks corresponding to tobacco alkaloids other than nicotine could be detected in the alkaloid thus isolated by a gas-liquid chromatography. Therefore, in this paper the content of alkaloids in $N$. tabacum determined by the above procedure is considered as that of nicotine.

The $N$. glutinosa plants harvested were separated into roots and aerial portions and blended separately with $80 \%$ ethanol containing $0.5 \%$ acetic acid in a Waring blender. The blended material was filtered and the filtrate was concentrated in a rotary evaporator under reduced pressure. The condensate was made strongly alkaline by addition of $6 \mathrm{~N}$ sodium hydroxide and then extrated 5 times by shaking with equal volumes of chloroform. The combined chloroform solution was extracted 3 times with $0.5 \mathrm{~N}$ hydrochloric acid. The resulting acid solution was evaporated to dryness and the residue was dissolved in $1 \mathrm{ml}$ of $0.01 \mathrm{~N}$ hydrochloric acid which contained appropriate amount of quinoline as a internal standard for gas chromatographic assay. Fifty microliters of the alkaloids solution thus obtained was submitted to a gas-liquid chromatography using the same apparatus as previously reported.6) The apparatus was equipped with a hydrogen flame ionization detector and a $4 \mathrm{~mm} \times 2.25 \mathrm{~m}$ stainless steel column packed with sodium hydroxide treated Shimalite (30 60 mesh) coated with $25 \%$ by weight silicon DC 550 . At $200^{\circ} \mathrm{C}$ with a $\mathrm{N}_{2}$ flow of $60 \mathrm{ml} / \mathrm{min}$.,

5) C. O. Willits, M. L. Swain, J. A. Connelly and B. A. Brice, Anal. Chem., 22, 430 (1950).

6) N. Yasumatsu and S. Akaike, J. Agr. Chem. Soc. Japan, 39, 347 (1965). 
the following retention times were obtained: quinoline, $11 \mathrm{~min} .30 \mathrm{sec}$, nicotine, $15 \mathrm{~min} .30 \mathrm{sec}$; nornicotine, $24 \mathrm{~min} .20 \mathrm{sec}$; anabasine, $30 \mathrm{~min} .30 \mathrm{sec}$; anatabine, $36 \mathrm{~min} .40 \mathrm{sec}$. Each of the alkaloids was determined from their relations to the internal standard, quinoline, in respect to peak areas and sensitivities.

Determination of nitrogen and ${ }^{15} \mathrm{~N}$ abundance in nitrogen. Nitrogen in the plant materials and the precipitate of nicotine silicotungstate was determined by Kjeldahl's method. Using the distillate made by Kjeldahl's method, ${ }^{15} \mathrm{~N}$ abundance in the ammonium-nitrogen was measured by a massspectrometer (Hitachi RMI-2).

Determination of protein-nitrogen, soluble sugars and starch. Powdered tobacco sample was boiled with $0.5 \%$ acetic acid for $5 \mathrm{~min}$, and filtrated. The residue was washed with the same solution. Nitrogen in the residue was determined by Kjeldahl's method and expressed as protein-nitrogen. Soluble sugars were extracted from the powdered sample with $80 \%$ ethanol, hydrolyzed by $4 \%$ sulfuric acid for 15 min. and determined by the method of Somogyi.7) The residue obtained by $80 \%$ ethanol extraction was hydrolyzed by $2.5 \%$ hydrochloric acid for 2 hrs. and after removal of protein sugars in the hydrolyzate were determined by the method above mentioned. Starch was calculated from the sugars thus determined.

\section{RESULTS AND DISCUSSION}

Effects of auxin addition to nutrient solution

Effects of IAA addition to nutrient solution at the concentration of $0,1,10$ and 100 p.p.m. were tested on $N$. tabacum plants. Little change in plant growth was observed by the treatment with 1 p.p.m. of IAA but leaves of the plants treated with up to 10 p.p.m. of IAA appeared to be darker green and exhibited bending or twisting, one of the characteristic symptoms of auxin treatment. Dry weight of the plants treated with 100 p.p.m. of IAA decreased (Fig. 1) and a marked decrease was observed in the roots. Nicotine content in the plants in dry weight basis was decreased with the increasing amount of IAA supplied and total nicotine in the plants treated with 100 p.p.m. of IAA

7) M. Somogyi, J. Biol. Chem., 195, 19 (1952)

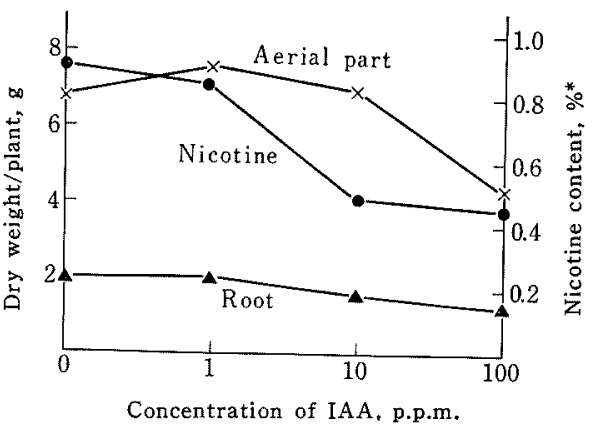

FIG. 1. Effects of IAA Added to Nutrient Solution upon Dry Weight and Nicotine Content of $N$. tabacum.

* Dry matter basis.

was reduced to about $30 \%$ of the control. A similar experiment was carried out using 2,4-D, one of the typical synthetic auxins, and the results are presented in Table $I$. Nicotine content of $N$. tabacum was reduced by the treatment with 1 p.p.m. of 2,4-D similarly as in the case of IAA.

Reduced nicotine content in tobacco plants may be attributed to a reduced nicotine production or an accelerated degradation of nicotine produced. To study the possible role of auxin treatment in nicotine production, ${ }^{15} \mathrm{~N}$-labeled nitrate was administrated to $N$. tabacum plants which were pretreated with 1 p.p.m. of 2, 4-D for $24 \mathrm{hrs} .{ }^{15} \mathrm{~N}$ found in total-nitrogen in the plants treated with $2,4-\mathrm{D}$ was lower than that of control (Table II), indicating that 2,4-D treated plant absorbed a smaller amount of nitrogen. Larger effects of the treatment were found in the decrease of ${ }^{15} \mathrm{~N}$ in nicotine and in the decreased proportion of nicotine $-{ }^{15} \mathrm{~N}$ to total $-{ }^{15} \mathrm{~N}$. These result may indicate that decrease in nicotine content of the plant treated with 2,4-D is resulted from decrease of nicotine production.

Effect of 2, 4-D treatment on the tobacco alkaloids other than nicotine was examined using Nicotiana glutinosa, which is known to have a suitable distribution of the various 
Table I. Effect of 2, 4-D Added to Nutrient Solution on Nicotine of $N$. tabacum

\begin{tabular}{|c|c|c|c|c|c|c|c|}
\hline \multirow{2}{*}{ Treatment } & \multicolumn{3}{|c|}{ Dry weight/plant $g$} & \multicolumn{3}{|c|}{ Nicotine content* $\%$} & \multirow{2}{*}{$\begin{array}{l}\text { Total nicotine } \\
\text { mg/plant }\end{array}$} \\
\hline & Leaves & Stem & Root & Leaves & Stem & Root & \\
\hline & 5.12 & 1.69 & 1.82 & 0.87 & 0.82 & 1.16 & 79.1 \\
\hline 2, 4-D & 5.37 & 1.54 & 1.73 & 0.43 & 0.67 & 0.80 & 47.1 \\
\hline
\end{tabular}

* Dry matter basis.

TABLE II. EFFeCT OF 2,4-D UPON THE INCORPORATION OF NITRATE-15N INTO NICOTINE OF $N$. tabacum

$\begin{array}{lccc}\text { Treatment } & \begin{array}{c}15 \mathrm{~N}-\text { Excess } \\ \text { in total-N } \\ \%\end{array} & \begin{array}{c}15 \mathrm{~N} \text {-Excess } \\ \text { in nicotine-N }\end{array} & \begin{array}{c}\text { Per cent of } \\ \text { nicotine-15N } \\ \text { in total-15N }\end{array} \\ \text { No addition } & 1.34 & 1.04 & 1.07 \\ \text { 2, 4-D } & 0.57 & 0.11 & 0.27\end{array}$

alkaloids. ${ }^{8 \sim 10)}$ The data in Table III reveal that 2, 4-D treatment decreased not only the content of nicotine but also that of nornicotine, anabasine and anatabine in both roots and aerial portions. From the study of the alkaloid content of grafted tomato and $N$. glutinosa, the most of nornicotine in mature $N$. glutinosa seems to be formed in the aerial portions by demethylation of nicotine translocated from the roots. ${ }^{11\}}$ Another experiment using nitrate- ${ }^{15} \mathrm{~N}$ indicated that nornicotine in $N$. glutinosa can also arise independently of nicotine. ${ }^{12}$ Therefore, decrease in nornicotine content of the plants may be attributed to the following three causes; the first is a reduced production of nicotine as a nornictine precursor, the second is a decreased nor- nicotine synthesis independent of nicotine, and the third is a inhibited demethylation of nicotine produced in the roots. From the result obtained it was impossible to decide the cause by which the nornicotine content in treated plants was decreased. Anabasin and anatabine contain piperidine and piperideine ring in their molecules, respectively. Biosynthetic studies of tobacco alkaloids have indicated that there are some differences among the synthetic pathways of anabasine, anatabine, and pyrrolidine alkaloids such as nicotine and nornicotine. ${ }^{13,14)}$ But Table III looks like to suggest that 2,4-D treatment reduces the contents of nicotine, nornicotine, anabasine and anatabine in $N$. glutinosa by affecting the common site of tobacco alkaloids biosynthesis where pyridine ring formation is concerned.

Effects of foliar application of synthetic auxins From the results mentioned above, it was obvious that alkaloid content of Nicotiana plants was decreased by the auxin absorbed from the roots where the most of the tobacco alkaloid was produced. However, if foliar

TABLE III. EFFECT of 2, 4-D UPON ALKALOID of $N$. glutinosa

$\begin{array}{clc}\text { Treatment } & \text { Plant part } & \begin{array}{c}\text { Fresh weight } \\ \text { g }\end{array} \\ \text { No addition } & \text { Aerial } & 42.4 \\ & \text { Root } & 23.5 \\ \text { Whole plant } & 65.9 \\ \text { 2, 4-D } & \text { Aerial } & 31.5 \\ \text { Root } & 19.2 \\ \text { Whole plant } & 50.7\end{array}$

8) T. C. Tso and R. N. Jeffrey, Plant Physiol., 31, 433 (1956).

9) E. Wada, T. Kisaki and M. Ihida, Arch. Biochem. Biophys., 80, 258 (1959).

10) W. L. Alworth and H. Rapoport, ibid., 112, 45 (1965).

\begin{tabular}{cccc}
\multicolumn{3}{c}{$\mu \mathrm{g}$ Alkaloid in $\mathbf{l g}$ of fresh matter } \\
Nicotine & Nornicotine & Anabasine & Anatabine \\
580 & 88.0 & 4.4 & 6.0 \\
360 & 15.8 & 10.4 & 8.3 \\
560 & 62.3 & 6.5 & 6.8 \\
240 & 10.1 & 1.4 & 3.6 \\
220 & 2.5 & 1.5 & 0.9 \\
230 & 7.2 & 1.4 & 2.6
\end{tabular}

11) R.F. Dawson, Am. J. Botany, 32, 416 (1945),

12) T. G. Tso, Arch. Biochem. Biophys., 92, 248 (1961).

13) E. Leete, J. Am. Chem. Soc., 78, 3520 (1956).

14) T. G. Tso and R. N. Jeffrey, Arch. Biochem. Biophys., 80, 46 (1959). 
application of auxin is as effective as the treatment in solution, it would be suitable for the practical purpose to regulate the alkaloid content in tobacco plants.

An experiment was planned to test the effectiveness of foliar application of eleven kinds of synthetic auxins on the nicotine content of $N$. tabacum. In this experiment each plant received a $20 \mathrm{ml}$ of 50 p.p.m. aqueous solution of the compound after decapitation of the plant. Beside the effect on nicotine formation in the plant, auxin treatment caused other effects such as bending or twisting of leaves, inhibited development of lateral buds, and adventitious root formation in stems. These results are summarized in Table IV. Nicotine content of the plants was decreased by any of the treatment but dry weight of the plants was not altered significantly. Larger effects on the nicotine content were obtained by the treatment of auxins which showed strong activities in the oat coleoptile cylinder test according to Åberg. ${ }^{151}$ A low effectiveness of IAA on nicotine production in spite of the strong activity in the oat coleoptile test may be explained by its instability in higher plants as reported by many workers.

A similar experiment was carried out using larger plants grown in pots containing 101 of nutrient solution. The plants were decapitated when they had reached the stage of growth where flower buds were becoming evident. After the decapitation each plant received a $70 \mathrm{ml}$ of 50 p.p.m. aqueous solution of NAA, 2,4-D or 2, 4,5-T and the plant growth was continued, without additional application of the compounds, following 25 days. The results of this experiment are summarized in Fig. 2. Dry weight of the stems was increased by foliar application of auxins but that of roots was decreased. Nicotine contents in the leaves, stems and roots were remarkably decreased by the treatment. As described, the relation between root growth and nicotine synthesis has been well known. Consequently, one of the causes which reduce the nicotine in treated plants

TABLE IV. EFFECT of Foliar Application of SyNThetic AuXins ON NICOTINE OF $N$. tabacum

\begin{tabular}{|c|c|c|c|c|c|}
\hline Compound & $\begin{array}{l}\text { Dry weight/plant } \\
\mathrm{g}\end{array}$ & $\begin{array}{c}\text { Relative nicotine } \\
\text { content in whole } \\
\text { plant } \\
\text { (No addition }=100 \text { ) }\end{array}$ & Bending of & $\begin{array}{l}\text { Root initiation } \\
\text { in stem }\end{array}$ & $\begin{array}{l}\text { Inhibition } \\
\text { of sucher } \\
\text { development }\end{array}$ \\
\hline No addition & 10.1 & 100 & - & - & - \\
\hline IAA & 11.2 & 71 & $+t+$ & + & - \\
\hline NAA & 11.8 & 45 & $++t$ & +++ & ++ \\
\hline NOXA & 10.9 & 60 & - & + & - \\
\hline TBS & 11.4 & 67 & - & ++ & - \\
\hline $\mathrm{CPA}$ & 10.9 & 58 & - & ++ & - \\
\hline $2,4-\mathrm{D}$ & 11.1 & 47 & + & +++ & $+t+$ \\
\hline $2,4-\mathrm{DP}$ & 9.7 & 45 & ++ & + & - \\
\hline MCP & 10.0 & 28 & $+t$ & +++ & +++ \\
\hline MCPP & 11.2 & 52 & $+t+$ & + & - \\
\hline $2,4,5-\mathrm{T}$ & 9.6 & 48 & + & +++ & +++ \\
\hline $2,4,5-\mathrm{TP}$ & 10.4 & 38 & + & + & + \\
\hline
\end{tabular}

Auxin activity is indicated by +++ if very high, ++ if medium, and + if only slight. - denotes an inactive compound. 


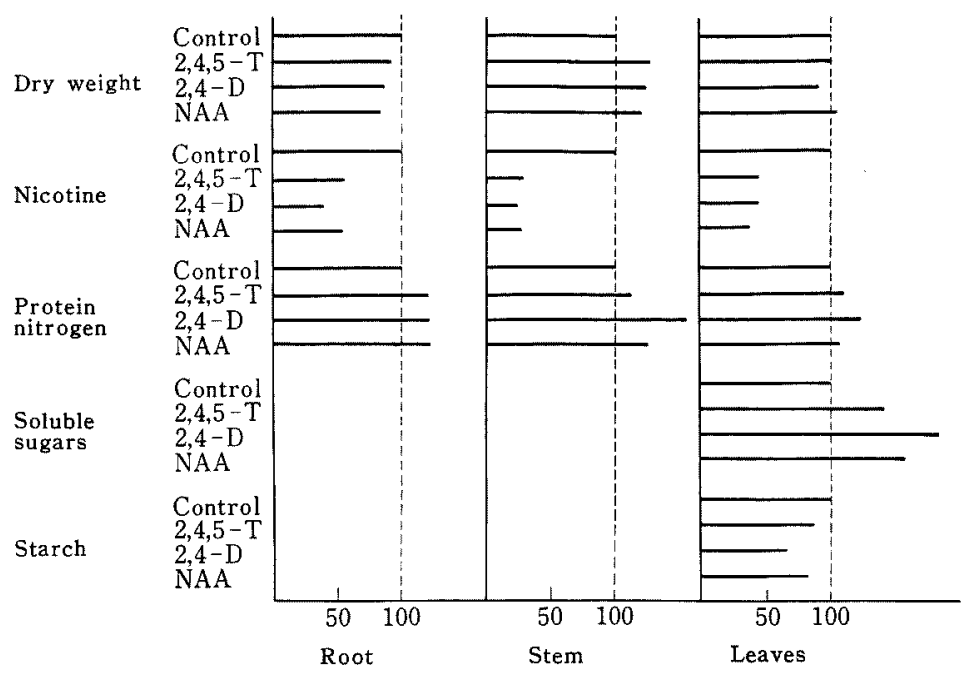

FIG. 2. Dry Weight and Relative Contents of Nicotine, Protein Nitrogen, Soluble Sugars and Starch in $N$. tabacum as Affected by Foliar Application of Synthetic Auxins.

Content of each component was determined in dry matter basis. The results are expressed as $\%$ of control.

may be attributed to the inhibition of root growth. Increase in the contents of protein in leaves, stems and roots and of soluble sugars in leaves were observed by the treatment but starch content in leaves was decreased. By the treatments of auxins the plant growth was not significantly influenced and thus the possibility of practical application of auxin to reduce the alkaloids content of commercial tabacco was suggested.

In the case of foliar application two possible site of auxin action may be considered. The first is the leaves where biosynthesis of nicotine precursor would be affected by the treatment and this reflect on the nicotine production in roots. The second is that of the roots where nicotine production would be directly influenced by the auxin which translocated from the leaves. If the latter is a valid site of auxin action, migration of applied auxin into the root may be essential to successful treatment. Such migration takes place via many processes, such as penetration into foliage and movement in a basipetal direction into the root. From the lipophilic nature of cuticle on the leaves it has mentioned that lipophilic compounds, such as the aliphatic esters of the phenoxy acids, penetrate the cuticle easily and that decreasing ionization enhances the penetration of 2, 4-D. ${ }^{161}$

To examine the effectiveness of different formulations of auxin, derivatives of CPA which seemed to be one of the suitable auxins for practical purpose were prepared and tested on $N$. tabacum. In this experiment a $15 \mathrm{ml}$ of $10^{-3} \mathrm{M}$ aqueous solution of the compounds was sprayed to a plant. Two kinds of salts and the amide were as effective as the free acid but the esters and methylamide were less effective (Table V). In another experiment 2, 4-D and 2, 4, 5-T were compared with their methylesters. Each plant was sprayed with $20 \mathrm{ml}$ of $2 \times 10^{-4} \mathrm{M}$ solution of

16) A. S. Crafts, "Encyclopedia of Plant Physiology XIV," Springer-Verlag, Berlin, 1961, p. 1044. 
Table V. Effect of Foliar Application of CPA DeRIVATIVes ON Nicotine of $N$. tabacum

$\begin{array}{lcccc}\text { Treatment } & \begin{array}{c}\text { Dry weight/plant } \\ \mathrm{g}\end{array} & \text { Leaf } & \text { Whole plant } & \begin{array}{c}\text { Nicotine content* } \\ \text { in whole plant } \\ \text { (No addition }=100 \text { ) }\end{array} \\ \text { No addition } & 12.36 & 1.27 & 0.98 & 100 \\ \text { CPA } & 13.30 & 0.45 & 0.39 & 43 \\ \text { CPA sodium salt } & 13.32 & 0.43 & 0.35 & 38 \\ \text { CPA diethanolamine salt } & 13.01 & 0.52 & 0.41 & 44 \\ \text { CPA amide } & 12.22 & 0.61 & 0.44 & 44 \\ \text { CPA methylamide } & 13.29 & 0.77 & 0.59 & 65 \\ \text { CPA methylester } & 14.41 & 0.55 & 0.46 & 55 \\ \text { CPA isopropylester } & 14.18 & 0.60 & 0.48 & 57\end{array}$

* Dry matter basis.

TABle VI. EfFects of Foliar Application of 2, 4-D, 2, 4, 5-T AND Their METhYLESTERS UPON NICOTINE OF $N$. tabacum

Compound

No addition

$2,4-\mathrm{D}$

2, 4-D methylester

$2,4,5-\mathrm{T}$

2, 4, 5-T methylester
Dry weight/Plant

14.4

14.0

14.0

13.6

13.6
Nicotine content*
$\%$

0.761

0.197

0.203

0.228

0.177
Relative nicotine content in whole plant

(No addition $=100$ ) 100

25

26

28

22

* Dry matter basis.

the compounds and the results are shown in Table VI. From the data in Table $\mathrm{V}$ and VI it is supposed that relative effectiveness of the methylesters to their free acids becomes greater with the increased number of substituted chlorine atoms in the molecules.

The present study revealed that exogenous auxin decrease the alkaloid biosynthesis in tobacco plants, but the mechanism of auxin

action remains to be solved. To elucidate this problem a biochemical study is in progress and it will be published elsewhere.

Acknowledgements. The author is indebted to Dr. D. Yoshida and Mr. T. Yamashita for helpful discussions and encouragement. Thanks are also due to Miss T. Mitake and Mrs. K. Yamaguchi for technical assistance. 